\title{
Revisión sistemática: Factores asociados al ausentismo laboral en países de América Latina
}

\author{
Systematic review: Factors associated with labor absenteeism in Latin American countries
}

Ruth Abigail Tatamuez-Tarapues ${ }^{*}$ orcid.org/0000-0003-1595-1020

Ana Milena Domínguez' orcid.org/0000-0003-2353-7292

Sonia Maritza Matabanchoy-Tulcán' orcid.org/0000-0002-3681-8440

1 Universidad de Nariño. San Juan de Pasto, Colombia

Fecha de recepción: Febrero 28 - $2018 \quad$ Fecha de revisión: Agosto 16 - $2018 \quad$ Fecha de aceptación: Diciembre 11 - 2018

Tatamuez-Tarapues RA, Domínguez AM, Matabanchoy-Tulcán SM. Revisión sistemática: Factores asociados al ausentismo laboral en países de América Latina. Univ. Salud. 2019;21(1):100-112. DoI: http://dx.doi.org/10.22267/rus.192101.143

\begin{abstract}
Resumen
Introducción: El ausentismo laboral ha sido entendido como la no asistencia al trabajo por un periodo de uno o más días laborales, considerado como una problemática de salud con un creciente interés por su impacto en la calidad de vida, salud mental del trabajador, economía y competitividad organizacional. Objetivo: Identificar factores atribuidos al ausentismo laboral en Países de América Latina en los últimos 20 años. Materiales y métodos: Se empleó la metodología de revisión sistemática, siguiendo las etapas de: construcción de la pregunta problema y objetivo; especificación de criterios de inclusión; formulación del plan de búsqueda; exploración de artículos; evaluación de calidad; sistematización de información; interpretación y presentación de resultados. Resultados: Se identificó que los factores atribuibles al ausentismo laboral se pueden agrupan en tres grupos: factores individuales, relacionados con las condiciones propias del trabajador, factores sociodemográficos y por último los intralaborales, que están conexos con el contexto laboral. Conclusión: La presencia del ausentismo laboral se debe a la confluencia de factores individuales, sociodemográficos, intralaborales y extralaborales, que se relacionan entre sí, corroborándose con la literatura que la presencia de este fenómeno se debe a una etiología multifactorial con efectos negativos a nivel individual y organizacional.
\end{abstract}

Palabras clave: Ausentismo; absentismo; ausencia. (Fuente: DeCS, Bireme).

\begin{abstract}
Introduction: Absenteeism has been understood as non-attendance at work for a period of one or more working days, which is considered as a health problem with a growing interest in its impact on the quality of life, mental health of the worker, economy and organizational competitiveness. Objective: To identify factors attributed to absenteeism in Latin American countries in the last 20 years. Materials and methods: The systematic review methodology was used following the stages of: construction of the problem question and objective; specification of inclusion criteria; formulation of the search plan; item browsing; quality assessment; systematization of information; interpretation and presentation of results. Results: The factors attributable to absenteeism can be grouped into three groups: individual factors related to the conditions of the worker, demographic factors and finally the intralabor ones, which are related to the labor context. Conclusion: The presence of labor absenteeism is due to the confluence of individual, demographic, intralabor and extralabor factors, which relate to each other. According to literature, the presence of this phenomenon is due to a multifactorial etiology with negative effects at the individual and organizational level.
\end{abstract}

Key words: Absenteeism; absenteeism; absence. (Source: DeCS, Bireme). 


\section{Introducción}

El ausentismo laboral es una problemática de salud pública que presenta un creciente interés de estudio por su impacto nocivo en la economía, la competitividad de las empresas y el desarrollo del talento humano, que afecta de forma global la salud mental del trabajador, pues interfiere en el despliegue óptimo de habilidades, conocimientos, destrezas, experiencias y aptitudes de quienes se consideran el capital intelectual de la organización y un elemento fundamental para el logro del éxito organizacional. Por esta razón, se hace necesario abordar el tema con el propósito de reconocer los factores con los cuales se relaciona el ausentismo ${ }^{(1)}$. Las investigaciones sobre este tema a nivel mundial son de gran relevancia en las organizaciones, dado que esta problemática ha incrementado en un $30 \%$ en los últimos 25 años, aumentando los costos empresariales en salubridad, interfiriendo en la productividad y ocasionando desgaste del recurso humano(2).

El concepto puntual y conciso, de la palabra ausentismo proviene del vocablo latino "absentis" la cual denota (ausente, separado, lejano) el abandono del puesto de trabajo y los deberes del mismo, incumpliéndose las condiciones establecidas en el contrato laboral(3). Por su parte, la Organización Internacional del Trabajo (OIT) ${ }^{(4)}$ define el ausentismo como la práctica realizada por un trabajador de no asistencia al trabajo por un período de uno o más días de los que se pensaba que iba a asistir, quedando excluidos los periodos vacacionales, las huelgas, períodos gestacionales y privación de la libertad. Además, la Organización Mundial de la Salud (OMS) ${ }^{(5)}$, hace alusión a este como la inasistencia "por causa directa o indirectamente evitable, tal como la enfermedad, cualquiera que sea su duración y carácter, así como las ausencias injustificadas durante toda o parte de la jornada laboral y los permisos circunstanciales dentro del horario laboral". Estos postulados concuerdan al referir que éste es la ausencia del trabajador a su lugar de trabajo en la jornada laboral, lo cual puede ser atribuido a diferentes factores justificados e injustificados, situación en la que se excluye circunstancias propias legales. Lo anterior permite extrapolar tres subcategorías del concepto, para comprender la esencia del mismo, las cuales son inasistencia al lugar de trabajo, tiempo de ausencia y causas generadoras al ausentismo.

El ausentismo se reconoce como un problema en todas las áreas del campo laboral dados los efectos negativos que conlleva al declive de la organización. La literatura reconoce dos tipos de ausentismo: el primero de ellos es aquel representado por la ausencia simple del trabajador a su lugar de trabajo. El segundo tipo, conocido como "cuerpo presente" o "presentismo laboral", que sucede cuando el trabajador, aunque no falta a su lugar de trabajo, no entrega su mejor desempeño en las actividades correspondientes, lo que conlleva a la disminución de su productividad(6).

En función de sus causas médicas, como convencionales y legales se clasifica en: primero, ausentismo legal o involuntario, el cual se caracteriza porque el trabajador continúa recibiendo remuneración. A esto se le podría denominar ausentismo retribuido y comprende, enfermedad normal, accidente laboral, licencias legales y enfermedad profesional. Segundo, ausentismo personal o involuntario, caracterizado porque el trabajador en dichas circunstancias no continúa recibiendo remuneración, que se podría denominar como ausentismo no retribuido y comprende permisos particulares, ausencias no autorizadas y conflictos laborales(7).

En este sentido, es preciso considerar una etiología multifactorial del ausentismo laboral, reconociendo la interacción de variables individuales, como la motivación, necesidades, valores, habilidades y conocimientos; variables de tipo ocupacional, como el tipo de empresa, la actividad a desempeñar, jornada laboral, métodos de producción y el tamaño de la planta laboral; y variables relacionadas con la organización, como el clima laboral de la empresa y las políticas institucionales. Por ello, es necesario reconocer que el principal tipo de ausentismo laboral es el relacionado con incapacidad médica por causas asociadas con la salud, por enfermedad o 
accidente. Teniendo en cuenta lo mencionado, el objetivo del presente estudio fue identificar qué factores o causas se le han atribuido al ausentismo laboral en países de América Latina en los últimos 20 años, con el propósito de contribuir a la visibilización de esta problemática, para que en próximas investigaciones se planteen estrategias encaminadas a impactar aquellos factores que lo ocasionan.

\section{Materiales y métodos}

\section{Tipo de estudio}

El estudio se llevó a cabo a partir de revisión sistemática de la literatura, el tipo de estudio fue de corte integrativo, observacional y retrospectivo.

\section{Estrategias de búsqueda}

Se revisó publicaciones e investigaciones sobre los factores o causas que se le atribuyen al ausentismo laboral en países de América Latina en las bases de datos SciELO, Redalyc, Dialnet, Open access Journal, Eric, Wiley Open Access, SSRN electronic Library y Ebsco Host.

\section{Criterios de selección}

Los criterios de inclusión que se tuvieron en cuenta para la búsqueda, se basaron en la relación de los objetivos de los artículos con los intereses planteados en este trabajo, teniendo en cuenta los siguientes criterios:

1. Artículos publicados en las bases de datos mencionadas anteriormente, entre los años 1997 a 2017.

2. Artículos desarrollados en países de América Latina

3. Escritura en idioma español, inglés y portugués

4. Artículo de investigación finalizada.

5. Referencias disponibles.

6. Acceso al texto completo

Para la búsqueda de los artículos se tuvo en cuenta las palabras clave: factores, causas, caracterización, ausentismo laboral, absentismo laboral, en inglés work absenteeism, absenteísmo. Para el presente artículo se retomaron exclusivamente estudios y artículos de bases de datos y revistas científicas con el propósito de garantizar la calidad del mismo, desarrollando el siguiente flujo de trabajo:

1) Construcción de la pregunta y objetivo de investigación;

2) Especificación de los criterios de inclusión de los artículos;

3) Formulación del plan de búsqueda de literatura;

4) Búsqueda de artículos en las bases de datos mencionadas;

5) Evaluación de la calidad de los estudios de acuerdo a criterios establecidos;

6) Sistematización de la información y

7) Interpretación y presentación de resultados.

\section{Resultados}

La revisión se centró en países de América Latina tomando bases de datos regionales debido a que la mayor producción científica se identificó en este contexto; por tanto, se encontró los siguientes resultados, que para una mayor comprensión, se agruparon de la siguiente manera:

\section{Año de publicación}

En principio se encontraron 73 artículos relacionados con ausentismo laboral, de los cuales se descartaron 23 debido a que no cumplían con los criterios de inclusión establecidos como: año de publicación, factores o causas del ausentismo laboral, desarrollo de la investigación en países de América Latina y visualización de todo el documento. El total de estudios realizados sobre ausentismo laboral fueron 50, considerando que durante los años 1997 hasta el 2004 no se realizaron publicaciones, por esto, del periodo 2005 a 2017 se perciben como relativamente actuales. En el año 2014 se publicaron 13 de los 50 estudios, en el 2012 se registran 6 publicaciones y en los años 2005 a 2010 se evidencia mínima publicación de estudios anuales.

En cuanto a los países de América Latina en donde se desarrollaron dichos estudios, se evidenció que la mayoría de las publicaciones se realizaron en Brasil, seguido de Colombia con un $42 \%$ y $34 \%$ 
respectivamente; en Chile con un 6\%, en Perú, Argentina y México se encontró publicaciones con un $4 \%$ para cada País y para Costa Rica, Uruguay y Venezuela con un 2\%.

Se reconoció que en 2012 incrementó el número de investigaciones, así: 4 en Brasil y una en Colombia y Costa Rica obteniendo un total de 6 investigaciones; en el año 2014 se registró 6 estudios en Brasil, 3 en Colombia, 2 en Chile, 1 en Argentina y 1 Uruguay para un total de 13 investigaciones. En el último año revisado 2017 se identificó 1 publicación en Brasil, 1 en Colombia y 1 en Perú.

\section{Sector de estudio}

Según la revisión, se identificó que la mayor parte de estudios fueron realizados en el salud(8-30) con un $46 \%$, seguido por el industrial(6,31-44), con un $30 \%$, educativo( ${ }^{(45-50)}$, con un $12 \%$, y finalmente, el sector público- ejecutivo(7,51-54), con un $12 \%$.

\section{Idioma de artículo}

Se identificó que la mayoría de los estudios se encuentran publicados en el idioma español con un 58\% dado que la búsqueda se realizó en países latinos, seguido del idioma inglés con un $8 \%$ y portugués con un $16 \%$, también se evidenció que existe una alta cantidad de estudios publicados en dos idiomas a la vez, inglés/portugués con un $16 \%$, y una cantidad mínima español/ portugués, español/ inglés con un $2 \%$ para cada grupo.

\section{Definición de ausentismo laboral}

Se visualizó que de los 50 artículos publicados, 30 (60\%) no refieren una definición precisa sobre ausentismo laboral, puesto que sus conceptos se asocian con los factores relacionados con el ausentismo. Los 20 restantes, para definir esta categoría, 10 estudios retoman la definición expuesta por la OIT, 1 la presentada por la OMS y 9 se basan en autores investigadores del tema (Tabla 1).

Teniendo en cuenta las definiciones retomadas para esta revisión, es importante resaltar los elementos en común de los cuales se pudo inferir subcategorías y que llevaron a concluir la esencia del concepto del ausentismo laboral. La primera de ellas, la inasistencia al lugar de trabajo, en la cual se concuerda al referir que es la no asistencia al lugar de trabajo sin previo aviso. La segunda está relacionada con las causas que generan ausentismo, en la que se resalta las condiciones médicas, familiares, sociales y culturales. La última hace alusión al tiempo de inasistencia al lugar de trabajo, que puede comprender horas, días o meses, aunque las consecuencias varíen dependiendo de su duración, se debe considerar como ausentismo. Para mayor claridad, en la Tabla 2 se presentan las definiciones frecuentes que utilizaron los autores cuando hacen mención a cada una de las subcategorías.

Tabla 1. Clasificación por definición

\begin{tabular}{|c|c|c|c|}
\hline Fuente & Definición & Frecuencia & $\%$ \\
\hline $\begin{array}{l}\text { Organización } \\
\text { Internacional } \\
\text { del Trabajo } \\
\text { (OIT) }\end{array}$ & $\begin{array}{l}\text { Define el ausentismo laboral como una práctica realizada por un trabajador de no } \\
\text { asistencia al trabajo por un periodo de uno o más días en los que se pensaba que iba a } \\
\text { asistir quedando excluidos los periodos vacacionales, las huelgas, periodos gestacionales } \\
\text { y privación de la libertad( }{ }^{(4)} \text {. }\end{array}$ & 10 & 20 \\
\hline $\begin{array}{l}\text { Organización } \\
\text { Mundial de la } \\
\text { Salud (OMS) }\end{array}$ & $\begin{array}{l}\text { El ausentismo hace alusión a un fenómeno de inasistencia por causas ya sean directa o } \\
\text { indirectamente evitables tales como enfermedad, cualquiera que sea su duración y } \\
\text { carácter, así como las ausencias injustificadas durante toda o parte de la jornada laboral } \\
\text { y los permisos circunstanciales dentro del horario laboral(5). }\end{array}$ & 1 & 2 \\
\hline $\begin{array}{l}\text { Autores } \\
\text { investigadores }\end{array}$ & $\begin{array}{l}\text { Ausentismo es la suma de tiempo en el cual los empleados de determinada organización } \\
\text { se encuentran ausentes en el trabajo, no siendo la ausencia motivada por desempleo, } \\
\text { enfermedad prolongada o licencia(9,42). } \\
\text { El ausentismo laboral representa las ausencias no programadas al trabajo, por faltas y } \\
\text { licencias médicas. Las causas que llevan al ausentismo pueden estar relacionadas a varios } \\
\text { factores y ser clasificadas en factores de enfermedades, de trabajo, sociales, culturales, y } \\
\text { de personalidad(10,51). }\end{array}$ & 9 & 18 \\
\hline
\end{tabular}


El ausentismo laboral es la ausencia del trabajador de su lugar de trabajo y se produce por los retrasos, ausencias y faltas de los trabajadores en horario determinado para su jornada laboral(12).

Ausentismo entendido como la suma de los periodos en que, por cualquier motivo, los empleados se retardan o no asisten al trabajo en la empresa en momentos en que los empleados deben estar trabajando normalmente(19).

El ausentismo laboral se entiende como la falta de un trabajador en su lugar de trabajo por razones ajenas al desempeño del mismo(23).

El ausentismo es el incumplimiento de parte del empleado de sus obligaciones laborales, faltando al trabajo, cuando estaba previsto que se debía presentar, de manera justificada o injustificada, o no desarrollando sus tareas de forma voluntaria e involuntaria por: problemas médicos, obligaciones legales o familiares, etc., durante toda la jornada o una parte $^{(29,48)}$

Tabla 2 Definiciones de acuerdo a las subcategorías de ausentismo laboral

\begin{tabular}{|c|c|c|c|c|}
\hline 营 & 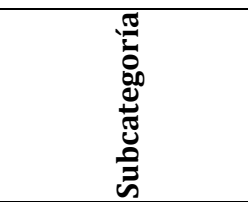 & Definiciones & 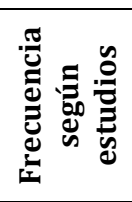 & a \\
\hline \multirow{10}{*}{ 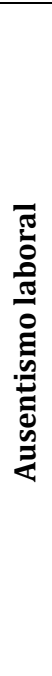 } & \multirow{3}{*}{$\begin{array}{l}\text { Inasistencia al } \\
\text { lugar de trabajo }\end{array}$} & $\begin{array}{l}\text { Conjunto de ausencias por parte de un trabajador de un determinado centro } \\
\text { de trabajo(51) }\end{array}$ & 3 & 6 \\
\hline & & $\begin{array}{l}\text { Es la no asistencia al trabajo por parte de un trabajador del que se pensaba } \\
\text { que iba a asistir(8) }\end{array}$ & 9 & 18 \\
\hline & & Ausencia del trabajador a su lugar de trabajo ${ }^{(48,12)}$ & 6 & 12 \\
\hline & \multirow{4}{*}{$\begin{array}{l}\text { Tiempo de } \\
\text { ausencia }\end{array}$} & $\begin{array}{l}\text { Es toda ausencia de su puesto de trabajo en horas que correspondan a un } \\
\text { día laborable dentro de la jornada legal de trabajo(8) }\end{array}$ & 3 & 6 \\
\hline & & Periodo de baja laboral atribuible a una incapacidad(49) & 3 & 6 \\
\hline & & $\begin{array}{l}\text { Periodo de } 1 \text { o más días (turnos) cuando ha sido asignado para un día de } \\
\text { trabajo(36) }\end{array}$ & 10 & 20 \\
\hline & & $\begin{array}{l}\text { Frecuencia o duración del trabajo perdido cuando los trabajadores no } \\
\text { asisten al trabajo(38) }\end{array}$ & 2 & 4 \\
\hline & \multirow{3}{*}{$\begin{array}{c}\text { Causas } \\
\text { generadoras de } \\
\text { ausentismo }\end{array}$} & $\begin{array}{l}\text { El ausentismo laboral es un síntoma que revela no sólo problemas del } \\
\text { trabajador, sino también del ámbito de la organización(15) }\end{array}$ & 2 & 4 \\
\hline & & $\begin{array}{l}\begin{array}{l}\text { Puede ser por causas médicas y no médicas, es decir, causas } \\
\text { multifactoriales(7) }\end{array} \\
\end{array}$ & 10 & 20 \\
\hline & & $\begin{array}{l}\text { Pueden estar relacionadas a varios factores, de trabajo, sociales, culturales } \\
\text { y de personalidad(12) }\end{array}$ & 2 & 4 \\
\hline
\end{tabular}

\section{Metodología utilizada para investigar ausentismo}

En la revisión se encontró diferentes tipos de investigación, entre ellos la metodología más empleada es la descriptiva $76 \%$, exploratoria con un $10 \%$, explicativa y correlacional con $8 \%$ y $6 \%$ respectivamente.

\section{Factores atribuidos al ausentismo laboral}

Se encontró que en la mayoría de las investigaciones los factores que causan el ausentismo, se logran fusionar en tres grupos, a la primera, se le asignó el nombre de factores individuales por su relación con estos, en los que se encuentra una relación directa con la salud del trabajador, como enfermedades del sistema osteomuscular, osteoarticular, del sistema conjuntivo, enfermedades del sistema respiratorio, enfermedades gastrointestinales, enfermedades infecciosas, traumatismos y del sistema circulatorio. También, se evidenció la relación con trastornos mentales y del comportamiento, entre ellos se destaca la relación con la ansiedad, depresión y estrés, reconociendo 
que cualquier tipo de afección a la salud genera incapacidad médica que en su mayoría tiende a extenderse. Según Mora et al., en la presencia de dichos trastornos mentales genera una incapacidad médica que ocasiona en promedio 35 días ausentes ${ }^{(8)}$.

Un segundo grupo hace alusión a variables sociodemográficas, en las que se identificó una relación con el sexo femenino, siendo este el más propenso a estar ausente por la responsabilidad familiar a su cargo(12); edad mayor o igual a 45 años, puesto que conforme aumenta la edad se incrementa la frecuencia del ausentismo de mediano y largo plazo(40); nivel de escolaridad, donde el trabajador de nivel técnico los que se ausentan con mayor frecuencia; estado civil siendo los solteros quienes se ausentan más de su lugar de trabajo; fuente de ingresos, considerando que el aumento de los ingresos por otras fuentes se relaciona con más ausencia(13).

En cuanto al tercer grupo, hace referencia a los factores intralaborales o propios de la organización como la sobrecarga laboral, en la cual las cargas psíquicas relacionadas a la propia naturaleza del trabajo y a los mecanismos de control y supervisión, son considerados como generadores de estrés y depresión que conllevan a ausentarse de su lugar de trabajo(10); factores ergonómicos e influencia de factores de riesgo en el sitio de trabajo, tales como espacio físico del trabajo, ambiente térmico, posturas de trabajo, son situaciones que influyen para que el trabajador se ausente de su lugar de trabajo; la insatisfacción laboral, donde los trabajadores perciben disminuidas sus posibilidades de expansión y crecimiento profesional, observando condiciones desfavorables e inadecuadas relaciones con los compañeros de trabajo que genera malestar ocasionando días de ausencia; enfermedades laborales que se producen por el ejercicio de una actividad laboral o por la exposición a diferentes agentes; cargo desempeñado, identificándose que en el equipo asistencial en casos de institución de salud tiende a ausentarse más que los administrativos; y jornada laboral, siendo los turnos nocturnos y matutinos e introducción de horas de trabajo flexible(12) lo que producen mayor ausencia. Lo mencionado coincide con la información encontrada en el $46 \%$ de las investigaciones revisadas que corresponden al sector salud; además, este tercer grupo de factores intralaborales se presenta en el 30\% de los estudios del sector industrial y en el $12 \%$ del sector educativo.

Teniendo en cuenta lo anterior, se identificó que en la mayoría de los estudios los factores influyentes para el ausentismo son múltiples para cada estudio, como se observa en la Tabla 3.

Tabla 3. Factores atribuidos al ausentismo encontrados en las investigaciones

\begin{tabular}{|c|c|c|}
\hline $\begin{array}{c}\text { Factores encontrados en las } \\
\text { investigaciones }\end{array}$ & Frecuencia & $\%$ \\
\hline Individuales & 10 & 20 \\
\hline $\begin{array}{l}\text { Individuales y } \\
\text { sociodemográficos }\end{array}$ & 13 & 26 \\
\hline Individuales e intralaborales & 5 & 10 \\
\hline $\begin{array}{l}\text { Individuales, sociodemográficos } \\
\text { e intralaborales }\end{array}$ & 8 & 16 \\
\hline $\begin{array}{l}\text { Individuales, sociodemográficos } \\
\text { y otras variables }\end{array}$ & 1 & 2 \\
\hline Individuales y otras variables & 2 & 4 \\
\hline Intralaborales & 2 & 4 \\
\hline Intralaborales y otras variables & 3 & 6 \\
\hline $\begin{array}{l}\text { Sociodemográficos e } \\
\text { intralaborales }\end{array}$ & 1 & 2 \\
\hline $\begin{array}{l}\text { Sociodemográficos, } \\
\text { intralaborales y otras variables }\end{array}$ & 2 & 4 \\
\hline $\begin{array}{l}\text { Individuales, intralaborales, } \\
\text { sociodemográficos y otras } \\
\text { variables }\end{array}$ & 1 & 2 \\
\hline Otras variables & 2 & 4 \\
\hline
\end{tabular}

\section{Relación del ausentismo con otras variables}

En la mayoría de los estudios revisados existe mayor relación con los factores individuales, seguido por los factores sociodemográficos e intralaborales, aclarando que existen investigaciones en las cuales se aborda los tres factores atribuibles al ausentismo. Es importante destacar que, en una mínima cantidad de estudios, se evidencia una relación con otras variables que abarcan factores como rasgos de personalidad(47), problemas relacionados con la salud bucal(36,52), índice de masa corporal(35,40), y responsabilidad 
familiar $^{(19,42)}$ (quienes no tienen hijos se ausentan con mayor ocurrencia).

\section{Discusión}

A partir de la revisión realizada, se reconoce que el ausentismo laboral es una problemática de salud pública con un reciente interés de estudio en América Latina desde el año 2005 en países como México. Así mismo, en el año 2008, en Brasil se dio inicio a investigaciones sobre ausentismo, evidenciando un incremento de producción investigativa en el año 2014 dando continuidad al estudio de esta temática hasta el momento. Si bien la mayor parte de las investigaciones revisadas se han realizado en países latinos, no se excluye el aporte de literatura construida en países de otros continentes para el enriquecimiento de este estudio. Puesto que esta problemática ha sido catalogada como un fenómeno que genera consecuencias negativas directas e indirectas en las organizaciones, en términos de costos, productividad, desgaste administrativo y de recurso humano(12). De tal manera que al elevarse el índice de ausentismo de los trabajadores de la empresa, automáticamente se reduce la calidad de la prestación del servicio y por ende la productividad y rentabilidad(55). A esto, se suman los resultados de investigaciones como esta, que muestran un gran interés y necesidad de estudiar las causas atribuibles al ausentismo laboral como una forma de contribuir a su abordaje y manejo.

Con respecto al sector donde se realiza mayor investigación frente a esta temática, la revisión permite visibilizar que el $38 \%$ de los estudios fueron desarrollados en instituciones de salud. Algunos autores ${ }^{(10)}$ refieren que los profesionales de la salud merecen mayor atención, puesto que otros resultados investigativos, hacen alusión que esta problemática apunta a altos índices de ocurrencia en este sector. Además, reconocen que los trabajadores del área asistencial sufren diferentes agravios a su salud, en los que se evidencia procesos de desgaste biopsíquico a los que están sometidos los trabajadores, sea por la propia naturaleza del trabajo como movimientos rápidos o repetitivos, esfuerzos excesivos y concentración de fuerzas mecánicas, posturas incorrectas sin neutralidad o por las condiciones en las que se realiza(12,56). Dado lo anterior, se infiere que el estudio del ausentismo laboral en instituciones de salud se torna necesario, por cuanto este recurso humano es el responsable de atender procesos de salud - enfermedad.

De acuerdo a esta revisión se identificó que en los cincos sectores abordados, las definiciones en su mayoría son propias respecto a las investigaciones; por esto, se establece la necesidad de comprender el por qué la construcción de la definición no es acogida de autores investigadores del tema u organizaciones, pues requieren un abordaje de aquellos factores que para dicha población de estudio, son determinantes para la presencia del ausentismo.

En consecuencia, se encuentra que el $60 \%$ de estos no refieren una conceptualización de ausentismo laboral, identificándose que, si bien no acogen una definición clara, sus conceptos hacen alusión a los factores a los cuales se les ha atribuido la ausencia de los trabajadores a su lugar de trabajo. El porcentaje restante de los estudios retoman la definición suministrada por la OIT, OMS y por autores investigadores del tema. Por lo anterior, es posible inferir subcategorías debido a que retoman elementos en común en sus definiciones, tales como: inasistencia al lugar de trabajo, tiempo de ausencia y causas generadoras de ausentismo. Lo anterior permite tener claridad y concordancia acerca del fenómeno para poder abordarlo, categorizarlo y analizarlo en relación al objetivo de estudio del presente.

Por otro lado, la metodología de estudio empleada en las diferentes investigaciones son en su mayoría de cohorte descriptivo y exploratorio, en menor medida estudios explicativos, reconociendo que estos últimos tienen como propósito ir más allá de la descripción de conceptos o fenómenos, pues van dirigidos a responder a las causas de los eventos físicos y sociales, su interés se centra en explicar el por qué ocurre un determinado fenómeno y en qué condiciones se da(57); en contraste a lo referido, el ausentismo siendo una problemática para la salud pública y las organizaciones se muestra a partir de la revisión, con una etiología multifactorial pues los efectos que causan el ausentismo varían 
en su poder predictivo(23), además se relacionan variables influyentes no manipulables y difíciles de controlar, lo que hace que estudiar el fenómeno sea complejo, pues se ve interferido por las dinámicas propias del contexto y la generación de altos costos al tratar de intervenir las variables extrañas.

En lo relacionado a los factores atribuibles al ausentismo, Mora, et $a l^{(8)}$., refiere que el ausentismo laboral contiene una etiología multifactorial en la cual interaccionan diversos factores que generan la presencia de este fenómeno y conlleva a efectos negativos a nivel individual y organizacional. En concordancia con los resultados encontrados se corroborar que el ausentismo laboral no posee un solo factor determinante, ya que puede estar influenciado por factores propios del contexto organizacional, individuales y sociodemográficos los cuales se relacionan entre sí.

Por tanto, los factores que influyen en la presencia del ausentismo, se logran reunir en tres grandes grupos, en principio se reconoce los factores individuales con enfermedades generales del sistema osteomuscular, osteoarticular, del sistema conjuntivo, enfermedades del sistema respiratorio, gastrointestinales, infecciosas, traumatismos, del sistema circulatorio, enfermedades mentales y del comportamiento. En concordancia a los resultados de Trinidade et al., autores ${ }^{20}$ refieren que la principal causa de ausentismo es la atribuida a incapacidad médica de mediano y largo plazo por situaciones asociadas con la salud del trabajador; bien sea por enfermedad o accidente, condición que ocupa las tres cuartas partes del ausentismo total en las empresas donde se ha estudiado el fenómeno. Frente a esto, se infiere que el cuidado de la salud de cada trabajador forma parte primordial al momento de desarrollar una actividad laboral, puesto que ésta influirá en gran medida en la presencia o no del ausentismo. Por ello, es preciso que las instituciones reconozcan la importancia de generar acciones de promoción y prevención dentro de la organización, con el propósito de sensibilizar a los trabajadores frente a la necesidad de preservar su salud para un despliegue óptimo de sus capacidades.
En cuanto al segundo grupo, se hace alusión a los factores sociodemográficos, que, según los estudios revisados el ausentismo puede estar influenciado por la edad, evidenciándose en estos que, a mayor edad, mayor posibilidad de ausentismo; de igual manera, tanto el sexo femenino, el bajo nivel de escolaridad y el estado civil, que influyen en el ausentismo laboral. En relación a estos factores, Sánchez ${ }^{(2)}$ refiere que los estudios de ausentismo laboral son discrepantes, debido que algunos han demostrado que a mayor edad, mayor tasa de ausentismo, el cual es asociado a la carga de enfermedad; en contraposición a esto, dos estudios ${ }^{(34,50)}$ reconocen que con la edad entran en juego los factores motivacionales, demostrando que ésta, representada en mayor antigüedad de vinculación a la empresa, es un factor limitante para el ausentismo, por cuanto el trabajador siente que "debe" conservar su empleo hasta el momento de su jubilación. Si bien existen estas dos posturas, en la mayoría de documentación revisada se corrobora una relación directa entre la edad y el ausentismo (a mayor edad, mayor ausentismo), la información frente al otro postulado es mínima.

Con relación al tercer grupo, el ausentismo se relaciona con factores intralaborales o propios de la organización tales como sobrecarga laboral(30,31), insatisfacción(38,21,23), factores de riesgo en el sitio de $\operatorname{trabajo}^{(12)}$, cargo desempeñado(13,22,32) y jornada laboral $(13,20)$. En este sentido, las investigaciones demuestran que la organización estructural de una empresa y la asignación de roles, responsabilidades, poder y jerarquías, pueden influenciar de manera positiva o negativa en las motivaciones de los trabajadores. Es por esto, que los factores psicosociales cada vez adquieren un peso importante dentro de los factores determinantes del ausentismo; la alta demanda psicológica, la discriminación, el desequilibrio entre la vida personal y laboral, la baja probabilidad de tener crecimiento profesional dentro de la empresa, son sólo algunos de los ejemplos que condicionan las ausencias(2); es así, como la presencia de aquellos factores pueden ser un indicador de tensión en la 
relación hombre- trabajo- y disfunciones estructurales de la empresa(58,59).

Desde este punto de vista, es primordial reconocer al trabajador como parte elemental de la organización, tener presente sus aportes en las decisiones empresariales, promover la motivación, el entusiasmo y permitir el desarrollo de sus competencias profesionales, lo cual contribuirá a desarrollar un sentido de pertenencia con la organización, mejorar el ambiente laboral y las relaciones interpersonales que tendrán como resultado asegurar la permanencia del trabajador en su lugar de trabajo, cumpliendo adecuadamente sus funciones.

\section{Conclusiones}

El ausentismo laboral al ser una problemática de salud pública se ha ido estableciendo como un elemento de estudio de gran relevancia por las consecuencias que genera en la calidad de vida, en la salud mental del trabajador, en la economía y competitividad de la organización, razón por la cual el interés en los últimos 20 años se ha centrado en identificar las situaciones a las cuales se le atribuye la presencia de esta problemática, con el propósito de más adelante lograr intervenir de manera integral esta situación.

Esta investigación permite ratificar la teoría que refiere que la presencia del ausentismo laboral es atribuido a múltiples factores. Los cuales se agruparon en individuales, sociodemográficos e intralaborales, dado que para los países de América Latina presentan similitud en sus características al momento de determinar la presencia de este fenómeno. Sin embargo, es preciso recordar que el contexto en el cual se estudie dicha problemática influye de manera significativa en la presencia de unos factores $u$ otros.

Finalmente, el aporte del estudio al emplear la metodología de Revisión Sistemática, permite dar cuenta de un bagaje investigativo de años atrás y facilita al lector una información histórica con la integración de hallazgos de forma detallada de estudios primarios que examinaron los factores asociados al ausentismo laboral.

\section{Recomendaciones}

Para posteriores revisiones es preciso que el grupo investigativo pueda abordar mayor cantidad de bases de datos, incluyendo las de acceso restringido, con la finalidad de obtener mayor cobertura de la información frente a la problemática que se aborda e inclusión de los países latinos que no se lograron reconocer para el presente.

Finalmente, se invita a los interesados a dar continuidad al estudio del ausentismo laboral, centrándose en precisar tasa, frecuencia y días perdidos del ausentismo en las organizaciones con el propósito de comprender a profundidad este fenómeno.

\section{Conflicto de intereses}

Ninguno a declarar por los autores.

\section{Referencias}

1. Prieto-Bejarano PG. Gestión del talento humano como estrategia para retención del personal [Trabajo de grado especialista]. Medellín: Universidad de Medeillin; 2013 [citado 7 de marzo de 2018]; Disponible en: http://repository.udem.edu.co/handle/11407/160

2. Sánchez D. Ausentismo laboral: una visión desde la gestión de la seguridad y la salud en el trabajo. Revista Salud Bosque. 2015;5(1):43-54. Disponible en: http://m.uelbosque.edu.co/sites/default/files/publicac iones/revistas/revista_salud_bosque/volumen5_numer o1/07-articulo5-salud-bosque_vol5-No1.pdf

3. Camarota R. El ausentismo laboral de causa médica en la policía nacional uruguaya. Biomedicina. 2015;10(1):34$53 . \quad$ Disponible en: http://www.um.edu.uy/docs/ausentismo_laboral.pdf ISSN 1510-9747

4. Organización Internacional del Trabajo [Internet]. Ginebra: OIT; 2018. [Citado 7 de marzo de 2018]. Disponible en: http://www.ilo.org/global/lang-es/index.htm

5. Organización Mundial de la Salud. Programas y proyectos [Internet]. Ginebra: OMS; 2018. [citado 7 de marzo de 2018]. Disponible en: http://www.who.int/es/

6. Rovida S, Adas T, Martins RJ, Garbin Í, José A, Garbin S, et al. Absentismo laboral por patología odontológica y médica en una industria de gran porte del sector mecánico y mueblero. Medicina y Seguridad del Trabajo. 2014;60(236):489-96. Disponible en: http://scielo.isciii.es/pdf/mesetra/v60n236/original2. pdf 
7. Escobar-Aramburo MF, Duarte-Suárez ML, CaicedoCampo LL, García MM, Valderrama-Aguirre A, Cruz AM. Ausentismo Laboral por Enfermedad de Origen Infeccioso en una Institución Forense. Revista Colombiana de Salud Ocupacional. 2015;3(2):12-7. Disponible en: http://revistasojs.unilibrecali.edu.co/index.php/rcso/a rticle/view/88/98 ISSN: 2322 634X

8. Mora DM, Mejía Z, Rincón E, Barrios RM, Padilla FR. Ausentismo laboral de causa médica en el Instituto Autónomo Hospital Universitario de Los Andes. MedULA: revista de la Facultad de Medicina. 2006;14(1):22-6. Disponible en: http://www.saber.ula.ve/bitstream/handle/12345678 9/21861/articulo3.pdf;jsessionid=CC3FE0E01B099100 A7F6E3D16A3C2BA6? sequence $=2$

9. Becker SG, Oliveira MLC de. Study on the absenteeism of nursing professionals in a psychiatric center in Manaus, Brazil. Revista Latino-Americana de Enfermagem. 2008;16(1):109-14. Disponible en: http://www.scielo.br/scielo.php?pid=S0104$11692008000100017 \&$ script=sci_abstract\&tlng=es ISSN 1518-8345. http://dx.doi.org/10.1590/S010411692008000100017

10. Sancinetti TR, Gaidzinski RR, Felli VEA, Fugulin FMT, Baptista PCP, Ciampone MHT, et al. Absenteeism disease in the nursing staff: relationship with the occupation tax. Revista da Escola de Enfermagem da USP. 2009;43(SPE2):1277-83. Disponible en: http://www.scielo.br/scielo.php?script=sci_arttext\&pi $\mathrm{d}=$ S0080-62342009000600023 ISSN 1980-220X http://dx.doi.org/10.1590/S008062342009000600023

11. Reis RJ dos, Rocca L, Freitas P de, Silveira AM, Bonilla L, Mercedez I, et al. Factors related to sickness absenteeism among nursing personnel. Revista de Saúde Pública. 2003;37(5):616-23. Disponible http://www.scielo.br/scielo.php?pid=S003489102003000500011\&script=sci_arttext\&tlng=en ISSN 1518-8787. http://dx.doi.org/10.1590/S003489102003000500011

12. Díaz M del CM, Marziale MHP, Robazzi ML do CC, Freitas FCT de. Lesiones Osteomusculares En Trabajadores De Un Hospital Mexicano Y La Ocurrencia Del Ausentismo. Ciencia y Enfermería. 2010;XVI(2):35-46. Disponible en: https://scielo.conicyt.cl/scielo.php?script=sci_arttext\& pid=S0717-95532010000200005 ISSN 0717-9553. http://dx.doi.org/10.4067/S071795532010000200005

13. Ávila SM, León DM. Problemática en una central de urgencias de institución de salud de primer nivel debida al ausentismo del personal por incapacidad médica. Cuadernos Latinoamericanos de Administración. 2011;VII(13):45-55. Disponible en: http://bibliotecadigital.udea.edu.co/bitstream/10495/ 5539/1/CuelloKatherine_2016_AusentismoCausaMedic a.pdf

14. Ferreira EV, Amorim MJ, Lemos R, Silva FO, Filho J. Absenteismo dos trabalhadores de enfermagem em um hospital universitario do estado de Pernambuco. Rev Rene, Fortaleza [Internet]. 2011 [citado 7 de marzo de
2018];12(4):742-9. Disponible en: https://www.researchgate.net/publication/26827034 7_ABSENTEISMO_DOS_TRABALHADORES_DE_ENFERM AGEM_EM_UM_HOSPITAL_UNIVERSITARIO_DO_ESTAD O_DE_PERNAMBUCO

15. Ferreira RC, Härter-Griep R, Mendes MJ, Rotenberg L. Abordagem multifatorial do absenteísmo por doença em trabalhadores de enfermagem [Internet]. Revista de Saúde Pública. 2012 [citado 7 de marzo de 2018];46(2):259-268. Disponible en: http://www.redalyc.org/articulo.oa?id=67240197008 ISSN: 0034-8910

16. Mininel VA, Felli VEA, Silva EJ, Torri Z, Abreu AP, Branco MTA. Cargas de trabalho, processos de desgaste e absenteísmo-doença em enfermagem. Revista LatinoAmericana de Enfermagem. 2013;21(6):1290-7. Disponible http://www.scielo.br/scielo.php?script=sci_arttext\&pi $\mathrm{d}=\mathrm{S} 0104-$

$11692013000601290 \& \operatorname{lng}=e n \& n r m=i s o \& \operatorname{lng}=p t \quad$ ISSN $1518-8345$.

1169.2992 .2366

17. Olivares-Faúndez VE, Gil-Monte PR, Mena L, JélvezWilke C, Figueiredo-Ferraz H. Relationships between burnout and role ambiguity, role conflict and employee absenteeism among health workers. Terapia Psicológica. 2014;32(2):111-20. Disponible en: https://scielo.conicyt.cl/pdf/terpsicol/v32n2/art04.pd f ISSN 0718-4808

18. Bargas EB, Monteiro MI. Fatores relacionados ao absenteísmo por doença entre trabalhadores de Enfermagem. Acta Paulista de Enfermagem. 2014;27(6):533-8. Disponible en: http://www.scielo.br/scielo.php?script=sci_abstract\&p id=S0103-

$21002014000600533 \& \operatorname{lng}=e n \& n r m=i s o \& \operatorname{lng}=p t \quad$ ISSN 1982-0194. http://dx.doi.org/10.1590/19820194201400087

19. Bonilla C, Espitia LMC, Florez AM, Barbosa LPM, Pardo M, Barbosa WGJ. Ausentismo laboral en el centro de atención médica inmediata Vista Hermosa I nivel, empresa social del Estado. Ciencia y Tecnología para la Salud Visual y Ocular. 2014;12(1):21-32. Disponible en: https://revistas.lasalle.edu.co/index.php/sv/article/vie w/2695/2429 https://doi.org/10.19052/sv.2695

20. Trindade L de L, Grisa CC, Ostrovski VP, Adamy EK, Ferraz L, Amestoy SC, et al. Absentismo en el equipo de enfermería en el ambiente hospitalario. Enfermería Global. 2014;13(36):138-46. Disponible en: http://scielo.isciii.es/scielo.php?script=sci_arttext\&pid $=$ S1695-61412014000400008 ISSN 1695-6141

21. Mazo DA, Barrera LM. Factores relacionados con el absentismo laboral por causa médica en el personal de enfermería, Fundación Clínica del Norte, 2013 - 2014. Revista CES Salud Pública. 2016;7(1):3-16. Disponible en:

http://revistas.ces.edu.co/index.php/ces_salud_publica /article/view/3280/2640 http://dx.doi.org/10.21615/cessp.7.1.1

22. Marques D, Pereira M, Souza C, Vila V, Almeida C, Oliveira E. Absenteeism - illness of the nursing staff of a 
university hospital. Revista Brasileira de Enfermagem. 2015;68(5):876-882. Disponible http://www.scielo.br/scielo.php?pid=S0034-

$71672015000500876 \&$ script $=$ sci_arttext\&tlng=en ISSN 1984-0446 7167.2015680516i

23. Mendoza-Llanos R. Insatisfacción laboral como predictor del ausentismo en un hospital público. Revista médica de Chile. 2015;143(8):1028-33. Disponible en: https://scielo.conicyt.cl/pdf/rmc/v143n8/art10.pdf

24. Rascón MSC, Olivera CC, Sánchez A, Ceballos BH, Pianesi ME, Malarczuk C, et al. Ausentismo laboral y factores de riesgo cardiovascular en empleados públicos hospitalarios. Acta Bioquímica Clínica Latinoamericana. 2016;50(1):37-44. Disponible en: http://www.scielo.org.ar/pdf/abcl/v50n1/v50n1a07.p df

25. Santana L, Sarquis L, Brey C, Miranda F, Felli V, Santana $\mathrm{L}$, et al. Absenteeism due to mental disorders in health professionals at a hospital in southern Brazil. Revista Gaúcha de Enfermagem [Internet]. 2016 [citado 7 de marzo de 2018];37(1):e53485. Disponible en: http://www.scielo.br/scielo.php?pid=S1983-

14472016000100416\&script=sci_arttext\&tlng=en ISSN 1983-1447. http://dx.doi.org/10.1590/19831447.2016.01.53485

26. Rubia-Heylmann N, Ascari RA, Fumagalli-Schafnunes J. Profissionais Da Equipe De Enfermagem Absenteistas Em Um Hospital Universitário Em Santa Catarina, Brasil. Brazilian Journal of Surgery \& Clinical Research. 3 de junio de 2015;11(3):186-7.

27. Charry LJ, Cortés MJ. Factores de ausentismo laboral por causa médica de los trabajadores oficiales de la Universidad Surcolombiana 2004-2008 [Tesis]. Neiva, Colombia: Universidad Surcolombiana; 2008. Disponible en: https://contenidos.usco.edu.co/salud/images/docume ntos/grados/T.G.Salud-Ocupacional/93.T.G-LeidyJohana-Solano-Maria-Jimena-Cortes-Rodriguez2008.pdf

28. Cuevas YS, García TV, Villa ME. Caracterización del ausentismo laboral en un centro médico de I nivel [Tesis]. Bogotá: Universidad del Rosario; 2011. Disponible en: http://repository.urosario.edu.co/bitstream/handle/1 0336/2834/22647541-2012.pdf

29. Jiménez FM. Condiciones que fomentan el ausentismo laboral en el personal de enfermería y su impacto financiero en una institución de salud de alta complejidad. Bogotá 2011 y 2012 [Tesis]. Bogotá, Colombia: Pontificia Universidad Javeriana; 2014. Disponible

en: https://repository.javeriana.edu.co/bitstream/handle/ 10554/12062/JimenezBeltranFlorMariela2014.pdf;seq uence $=1$

30. González A. Posibles causas que ocasionan ausentismo laboral en el personal de enfermería del Hospital Tránsito Cáceres de Allende - Período 2010 [Tesis]. Córdoba, Argentina: Universidad Nacional de Córdoba; 2010.

Disponible
http://lildbi.fcm.unc.edu.ar/lildbi/tesis/Gonzalez_adria na.pdf

31. Bueno AM. Factores psicosociales que inciden en el ausentismo laboral del personal administrativo Gamma Ingenieros S.A, Bogotá D.C. Revista Tendencias \& Retos. 2008;(13):259-60. Disponible en: http://repository.lasalle.edu.co/bitstream/handle/101 85/12929/T62.08\%20B644f.pdf?sequence=1

32. Simões MRL, Rocha ADM, Souza C. Fatores associados ao absenteísmo-doença dos trabalhadores rurais de uma empresa florestal [Internet]. Revista Latino-Americana de Enfermagem. 2012 [citado 7 de marzo de 2018];20(4):1-9. Disponible en: http://www.redalyc.org/articulo.oa?id=281423331012

33. Oenning NSX, Carvalho FM, Lima VMC. Indicadores de absenteísmo e diagnósticos associados às licenças médicas de trabalhadores da área de serviços de uma indústria de petróleo. Revista Brasileira de Saúde Ocupacional. 2012;37(125):150-8. Disponible en: http://www.scielo.br/scielo.php?pid=S0303-

76572012000100018\&script=sci_abstract\&tlng=pt ISSN 0303-7657. http://dx.doi.org/10.1590/S030376572012000100018.

34. Arias-Moreno P, Carvajal R, Cruz ÁM. Ausentismo en Trabajadores con Reintegro Laboral. Revista Colombiana de Salud Ocupacional. 2015;2(4):14-8. Disponible en: http://revistasojs.unilibrecali.edu.co/index.php/rcso/a rticle/view/76/445

35. Zúñiga RAA, Ordoñez ESG, Osorio C, Escudero N, LópezAlbán CA, Ramírez-Vélez R. Obesidad Abdominal Y Ausentismo Por Causa Médica En Una Empresa De La Industria Metalmecánica En Cali, Colombia. Revista Peruana de Medicina Experimental y Salud Pública. 2013;30(2):251-5. Disponible en: http://www.scielo.org.pe/scielo.php?script=sci_arttext \&pid=S1726-46342013000200015

36. Miotto MHM de B, Barcellos LA, Lopes ZV. Dor de dente como preditor de absenteísmo em trabalhadores de uma indústria de sucos da Região Sudeste do Brasil. Ciência \& Saúde Coletiva. 2013;18(11):3183-90. Disponible en: http://www.scielo.br/scielo.php?pid=S1413$81232013001100009 \&$ script=sci_abstract \&thng=pt ISSN 1413-8123. http://dx.doi.org/10.1590/S141381232013001100009.

37. Trespalacios V, María E. Absentismo laboral por causa médica en trabajadores del área operativa de una compañía de extracción de minerales en Colombia, 2011. Medicina y Seguridad del Trabajo. 2013;59(230):93$101 . \quad$ Disponible en: http://scielo.isciii.es/pdf/mesetra/v59n230/original5. pdf

38. Oenning NSX, Carvalho FM, Lima VMC. Fatores de risco para absenteísmo com licença médica em trabalhadores da indústria de petróleo. Revista de Saúde Pública. 2014;48(1):103-12. Disponible http://www.scielo.br/scielo.php?pid=S0034$89102014000100103 \&$ script=sci_abstract $\&$ tlng $=$ pt ISSN 0034-8910. http://dx.doi.org/10.1590/S00348910.2014048004609. 
39. Simões MRL, Rocha ADM. Absenteísmo-doença entre trabalhadores de uma empresa florestal no Estado de Minas Gerais, Brasil. Revista Brasileira de Saúde Ocupacional. 2014;39(129):17-25. Disponible en: http://www.scielo.br/scielo.php?pid=S0303-

76572014000100017\&script=sci_abstract\&tlng=pt

ISSN 0303-7657. http://dx.doi.org/10.1590/03037657000061112.

40. Velásquez CF, Palomino JC, Ticse R. Relación entre el estado nutricional y los grados de ausentismo laboral en trabajadores de dos empresas peruanas. Acta Médica Peruana. 2017;34(1):6-15. Disponible en: http://www.scielo.org.pe/scielo.php?script=sci_arttext \&pid=S1728-59172017000100002

41. Andrade RD, Junior F, Jose G, Capistrano R, Teixeira CS, Silva Beltrame T, et al. Absenteísmo na Indústria está Associado com o Trabalho em Turnos e com Problemas no Sono. Ciencia \&amp; trabajo. 2017;19(58):35-41. Disponible en: https://scielo.conicyt.cl/scielo.php?script=sci_arttext\& pid=S0718-24492017000100035 ISSN 0718-2449 .http://dx.doi.org/10.4067/S071824492017000100035

42. Trejoz AF, Heredia JE. Estudio del costo y principales causas del ausentismo laboral en las empresas afiliadas a la Indi seccional Risaralda - Quindío [Tesis]. Pereira: Universidad Tecnológica de Pereira; 2010. Disponible en:

http://repositorio.utp.edu.co/dspace/bitstream/handl e/11059/2022/658314T787.pdf

43. Orozco J, Yépez L. Causas de ausentismo laboral en una empresa comercializadora de prendas de vestir con sede en Medellín [Tesis]. Medellín, Colombia: Universidad de Antioquia; 2017. Disponible en: http://bibliotecadigital.udea.edu.co/bitstream/10495/ 7869/1/OrozcoJessica_2017_AusentismoLaboralComer cializadora.pdf

44. Herrera FC, González MA. Caracterización del ausentismo laboral por causas médicas en una empresa de telecomunicaciones a nivel nacional [Tesis]. Bogotá, Colombia: Universidad del Rosario; 2015. Disponible en: http://repository.urosario.edu.co/bitstream/handle/1 0336/10188/52027582-2015.pdf

45. F JFS, L EM. Factores asociados al ausentismo laboral por causa médica en una institución de educación superior. Revista Facultad Nacional de Salud Pública. 2007;25(1):32-9. Disponible en: http://www.scielo.org.co/scielo.php?script=sci_arttext \&pid=S0120-

$386 X 2007000100005 \& \operatorname{lng}=e s \& n r m=i s o \& t \operatorname{lng}=e s \quad$ ISSN 0120-386X

46. Martínez-López E, Saldarriaga-Franco JF. Inactividad Física y Ausentismo en el Ámbito Laboral. Revista de Salud Pública. 2008;10(2):227-38. Disponible en: http://www.scielo.org.co/scielo.php?script=sci_arttext \&pid=S0124-

$00642008000200003 \& \operatorname{lng}=\mathrm{es} \& \mathrm{nrm}=\mathrm{iso} \& \operatorname{tng}=\mathrm{es} \quad$ ISSN 0124-0064

47. Suárez AP, Moreno NP. Personalidad Y Ausentismo En Trabajadores De Servicios Generales De Una Universidad Pública. Revista Hacia la Promoción de la
Salud. 2010;15(1):29-40. Disponible en: http://www.scielo.org.co/pdf/hpsal/v15n1/v15n1a02. pdf ISSN 0121-7577

48. Marques SVD, Martins G de B, Sobrinho OC. Saúde, trabalho e subjetividade: absenteísmo-doença de trabalhadores em uma universidade pública. Cadernos EBAPEBR. 2011;9:668-80. Disponible en: http://www.redalyc.org:9081/articulo.oa?id=3232278 30012 E-ISSN: 1679-3951

49. Berrocal I, Cubero MJ, Rojas JC, Salazar N. Índices y estadísticas del absentismo laboral: Caso Universidad Nacional. Revista ABRA. 2012;32(45):1-20. Disponible en:

https://dialnet.unirioja.es/servlet/articulo?codigo $=479$ 2061 ISSN-e 2215-2997

50. Calderón DCS. Características y tendencia de la población ausentista por causa médica en una institución de educación superior, Bogotá, 2011-2013. Cuadernos Latinoamericanos de Administración. 2015;XI(21):51-60. Disponible en: http://www.redalyc.org:9081/articulo.oa?id=4096407 43005 ISSN: 1900-5016

51. Navarrete-Escobar A, Gómez-López VM, Gómez-García $R$, Jiménez-Soto $M$. Ausentismo laboral en una institución de seguridad social y factores relacionados. Revista Médica del Instituto Mexicano del Seguro Social. 2005;43(5):373-6. Disponible en: http://www.redalyc.org/articulo.oa?id=457745544002 ISSN: 0443-5117

52. Miotto MHM de B, Silotti JCB, Barcellos LA. Dor dentária como motivo de absenteísmo em uma população de trabalhadores. Ciência \& Saúde Coletiva. 2012;17(5):1357-63. Disponible en: http://www.redalyc.org:9081/articulo.oa?id=6302335 0029 ISSN: 1413-8123.

53. Hoffmeister L, Vidal C, Vallebuona C, Ferrer N, Vásquez P, Núñez G. Factores Asociados a Accidentes, Enfermedades y Ausentismo Laboral: Análisis de una Cohorte de Trabajadores Formales en Chile. Ciencia \&amp; trabajo. abril de 2014;16(49):21-7. Disponible en: https://scielo.conicyt.cl/scielo.php?pid=S071824492014000100005\&script=sci_arttext ISSN 0718$2449 . \quad$ http://dx.doi.org/10.4067/S071824492014000100005

54. Miotto MHM de B, Lima WJG, Barcellos LA, Miotto MHM de B, Lima WJG, Barcellos LA. Association between dental pain and absenteeism among public workers from Southeastern Brazil. Revista Dor. 2014;15(3):173$7 . \quad$ Disponible en: http://www.scielo.br/scielo.php?script=sci_arttext\&pi $\mathrm{d}=\mathrm{S} 1806-00132014000300173$ ISSN 1806-0013. http://dx.doi.org/10.5935/1806-0013.20140038

55. Dionne G, Dostie B. New Evidence on the Determinants of Absenteeism Using Linked Employer-Employee Data [Internet]. Rochester, NY: Social Science Research Network; 2005 jun [citado 7 de marzo de 2018]. Report No.: ID 742585. Disponible en: https://papers.ssrn.com/abstract=742585 http://dx.doi.org/10.2139/ssrn.742585

56. Ferraro C. Incidencia de ausentismo laboral y factores determinantes en el personal de enfermería del Hospital 
Zonal Especializado en Oncología «Luciano Fortabat» de Olavarría [Tesis]. Santa Fé, Argentina: Universidad Nacional del Rosario; 2016. Disponible en: http://capacitasalud.com/biblioteca/wpcontent/uploads/2016/07/TESIS-FINAL.pdf

57. Hernández S, Fernández C, Baptista M. Metodología de la investigación [Internet]. 5a ed. México: McGraw-Hill; $2010 . \quad$ Disponible en: https://www.esup.edu.pe/descargas/dep_investigacio n/Metodologia\%20de\%20la\%20investigaci\%C3\%B3n \%205ta\%20Edici\%C3\%B3n.pdf

58. Peña LK. Factores y efectos del ausentismo laboral en la empresa Acerías paz del Río S A: propuesta de mejoramiento [Tesis]. Sogamoso: Universidad Pedagógica y Tecnológica de Colombia; 2016. Disponible en:

https://repositorio.uptc.edu.co/bitstream/001/1705/ 1/TGT-388.pdf

59. Tavera M. Factores que influyen en el ausentismo laboral y su impacto en el clima organizacional [Tesis]. Bogotá, Colombia: Universidad Militar Nueva Granada; 2017. Disponible en: https://repository.unimilitar.edu.co/bitstream/10654/ 17172/1/MosqueraTaveraNancy2017\%20.pdf 